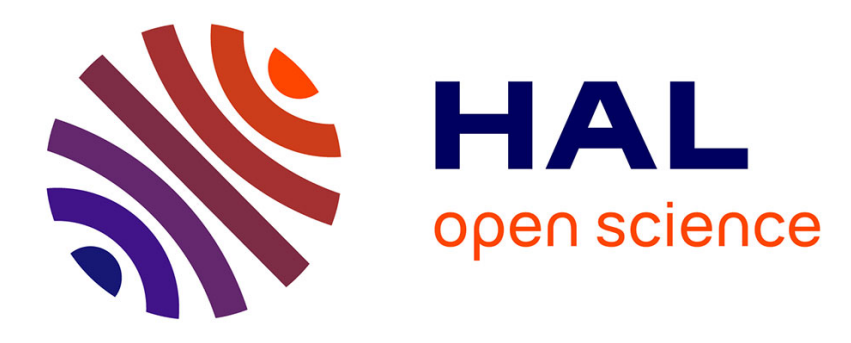

\title{
Les tragédies comme représentation de la langue française
}

\author{
Gilles Siouffi
}

\section{To cite this version:}

Gilles Siouffi. Les tragédies comme représentation de la langue française. sous la direction de G. Declercq et M. Rosellini,. Jean Racine 1699-1999, Actes du colloque du tricentenaire,, PUF, pp.415435., 2003. hal-03110142

\section{HAL Id: hal-03110142 \\ https://hal.science/hal-03110142}

Submitted on 14 Jan 2021

HAL is a multi-disciplinary open access archive for the deposit and dissemination of scientific research documents, whether they are published or not. The documents may come from teaching and research institutions in France or abroad, or from public or private research centers.
L'archive ouverte pluridisciplinaire HAL, est destinée au dépôt et à la diffusion de documents scientifiques de niveau recherche, publiés ou non, émanant des établissements d'enseignement et de recherche français ou étrangers, des laboratoires publics ou privés. 


\section{Gilles Siouffi}

«Les tragédies comme représentation de la langue française ", in Jean Racine 1699-1999, Actes du colloque du tricentenaire, sous la direction de G. Declercq et $M$. Rosellini, Paris, PUF, 2003, p. 415-435.

Si les tragédies de Racine peuvent être considérées comme une "représentation de la langue française", c'est tout d'abord bien sûr du fait qu'elles appartiennent au genre du théâtre : ainsi, elles donnent à entendre la langue, elles la mettent en scène, lui donnent corps.... Mais on peut aussi penser à une autre forme de "représentativité". Celle-ci engagerait, entre le texte lui-même et l'auditeur, ou tout du moins le récepteur de ce texte, une dimension linguistique intermédiaire dans laquelle ce qui est donné à entendre, ou à percevoir, acquiert un statut d'image, de tableau.

Notre objectif ici sera de s'interroger sur la manière dont, dans un espace laissé libre par la relative diminution 
de la pratique de l'éloquence après le début du XVIII ${ }^{\circ}$ siècle, la réception du texte des tragédies s'est trouvé engagée dans la voie d'une semblable recherche. Nous entendons ici essentiellement, bien évidemment, la réception de la langue de Racine.

Sous le rapport de la langue, il est clair que les enjeux qui ont touché le théâtre de Racine sont très différents de ceux qui touchèrent en son temps le théâtre de Corneille. Contrairement à Corneille, il apparaît en effet que Racine s'est rapidement trouvé placé dans une situation où son texte a été vu comme un objet privilégié par des commentateurs qui voulaient y retrouver, outre les marques d'un certain usage linguistique général qu'ils théorisaient et qu'ils préconisaient, les preuves de certains caractères intrinsèques de la langue française, qu'ils considéraient comme des éléments de son "génie". Indiscutablement, il s'agissait de grammairiens bien plus exigeants que ceux de la génération précédente : Boileau, Bouhours... ; de grammairiens, surtout, qui donnaient à chaque détail de langue, à chaque "minutie", comme on disait alors, qu'il s'agisse d'unions de termes, de constructions, de façons de parler, une signification propre à voir en eux la langue entière représentée. Ne disaient-ils pas de tel ou tel tour, sans autre forme de procès parfois, qu'il n'était "pas français" ? C'est ainsi qu'apparaissait Corneille, pour l'abbé de Villars ("tant de vers misérables, durs, sans pensée, sans tour, sans François, et sans construction", disait-il dans la Critique de Bérénice ${ }^{1}$ ).

1 voir Granet, Recueil de dissertations sur plusieurs tragédies de Corneille et de Racine, Paris, Gissey, 1740, p. 209. 
$\mathrm{Si}$, de Corneille, en 1660, on avait simplement attendu qu'il corrige les expressions dont l'usage avait trop évolué, ou qui ne paraissaient pas propres au genre tragique, de Racine, en revanche, on va attendre la perfection, on va attendre "le français". En 1818, Fontanier, l'auteur du Traité des Tropes, compile plus d'un siècle de commentaires sur Racine. Le titre et le sous-titre sont éloquents : "Etudes de la langue française sur Racine, ou Commentaire général et comparatif sur la diction et le style de ce grand classique, d'après[....] ; pour servir comme de cours pratique de langue française, et suppléer à l'insuffisance des grammaires, surtout en ce qui concerne l'application des principes." Racine fait donc figure d'auteur classique par excellence, de modèle de pureté. Entre temps, c'est souvent à des débats farouches que l'étude du texte des tragédies aura donné lieu, à des débats qui nous étonnent parfois aujourd'hui par leurs enjeux étranges, et qui nous semblent dépassés, tant les représentations ont réussi à faire de la langue racinienne une langue acceptable, plus même, une langue absolument belle, surplomb de toute pratique ultérieure, image d'une sorte de français mental échappant à l'histoire.

C'est donc Racine critiqué que nous lirons ici. Racine impitoyablement repris sur certains de ses plus beaux vers, accumulant les solécismes, les barbarismes, les fautes de langue comme un petit écolier, mais Racine sauvé..., finalement.

\section{Les étapes de la critique}

Retraçons donc brièvement, pour commencer, les principales étapes de la critique de langue sur Racine. 
La première étape est sans doute la Folle Querelle de Subligny, critique dialoguée d'Andromaque, et surtout la préface de cette petite pièce ${ }^{2}$. Dans cette comédie, après avoir longtemps disserté des questions générales, questions de type dramaturgique, esthétique, moral..., les personnages finissent par sortir le livre de leur poche et s'en lisent des extraits pour voir s'ils comprennent. Dans la préface, Subligny s'était fait fort d'avoir repéré plus de trois cents fautes dans Andromaque, pièce que par ailleurs, il trouve remarquable. Les personnages ne vont pas passer les trois cents fautes en revue, mais discutent néanmoins sur de nombreux détails ; dans la préface, par ailleurs, Subligny résume ses objections à la langue d'Andromaque. Ajourd'hui, le travail de Subligny nous paraît animé par un esprit de "scrupule" trop mécanique et trop impressionniste, sans véritable doctrine, -trop entièrement négatif aussi-, pour que nous puissions en retirer beaucoup de matière. Pour autant, deux faits notables ressortent de la lecture de cette critique. Tout d'abord quant à la position d'Andromaque dans le théâtre de Racine. Cela semble clair : Andromaque inaugure un nouveau système d'expression, une diction nouvelle, qu'on ne trouvait pas auparavant au théâtre. Certaines des qualités-et des défauts- qui seront plus tard abordés, sont déjà évoqués dans le commentaire de Subligny. D'autre part, la critique de Subligny est véritablement le signe qu'une pression grammaticale nouvelle, désormais, va s'exercer sur l'écriture des tragédies.

2 Citée dans Racine, Oeuvres complètes I, éd. G. Forestier, Bibliothèque de la Pléiade, 1999. 
La deuxième étape est constituée par un ensemble de commentaires peu attentifs au détail, globalement élogieux, et qui seront les commentaires décisifs dans la promotion $\mathrm{du}$ styliste. Quelques qualificatifs s'installent : SaintEvremond dans une lettre à $\mathrm{M}$. de Lionne à propos de Britannicus évoque les "vers magnifiques". Selon lui, il y a "du sublime"3. Dans le Parallèle de Monsieur Corneille et de Monsieur Racine de Longepierre (1686), on trouve plusieurs fois mentionné que le style de Racine est supérieur, plein de "grâce, de douceur, de délicatesse, de naturel, de tendresse" (Picard, 1956 : 157), termes qui rendent bien compte de l'évolution du goût en cette fin de siècle. Le parallèle de La Bruyère dans les Caractères (1688) 4 évoque le style "égal", "soutenu", parle de pièces "justes", "régulières", de "bon sens", il remarque les rimes "riches", "élégantes", "nombreuses", "harmonieuses", la simplicité de l'action. François de Callières, dans $\mathrm{Du}$ bel esprit, (1695), dit de Racine qu'il est "riche dans ses expressions, correct dans sa diction" (Picard, 1956 : 273). Tous ces commentaires témoignent d'une indéniable "classicisation" de la vision de Racine : en marge de la lecture détaillée, ils installent l'idée d'une langue emportant l'adhésion grâce à des qualités générales poussées à la perfection.

Au début du XVIII ${ }^{\circ}$ siècle cependant, donc après la mort du poète, on va à nouveau s'intéresser au détail grammatical des pièces, et ce d'une manière qui ne va pas sans rappeler Subligny. On sait que l'Académie avait un

3 J. Picard, Corpus Racinianum, Paris, Les Belles Lettres, 1956, p. 41.

4 La Bruyère, Les Caractères, Paris, Garnier, p. 88. 
projet de grammaire, projet devant compléter la réalisation du dictionnaire. On sait également que ce projet ne vit pas le jour, l'Académie ayant trop tardé et s'étant fait doubler par la grammaire "personnelle" de Regnier-Desmarais (1706). L'idée est alors venue de remplacer cette grammaire par un commentaire grammatical d'Athalie pour ce qui était de l'écriture poétique, et un commentaire de la traduction de Quinte-Curce par Vaugelas pour ce qui était de la prose. Pourquoi Athalie ? Parce que, dit l'Académie, "c'est une des plus parfaites tragedies que nous ayons" 5 . Ce choix illustre bien la manière dont le texte de Racine en vient à faire figure d'emblème, et se trouve en position de constituer un support pour une sorte de "grammaire pratique" du français. En réalité, ce commentaire, réalisé vers 1720 , ne parut qu'en 1807 dans l'édition de Racine faite par La Harpe 6 . Il est intitulé : "Sentimens de l'Académie sur Athalie", sur le modèle des célèbres Sentiments sur le Cid. Aujourd'hui, il décevra, car il très court, très laconique et peu argumenté.

Le premier grand commentaire grammatical du texte de Racine sera donc celui de D'Olivet, dans lequel on trouvera commentées toutes les pièces sauf Athalie, puisque celle-ci devait être étudiée par l'Académie ${ }^{7}$. Il en existe deux versions : celle de 1737, linéaire, qui suit les pièces acte par acte, scène par scène ; et celle de 1767, incluse

5 Les Registres de l'Académie Française, 3 vol. FirminDidot, 1895, II, p. 79-80.

6 Voir Les Remarques de l'Académie française sur le QuinteCurce de Vaugelas, éd. Wendy Ayres-Bennett et Philippe Caron, Paris, Presses de l'Ecole Normale Supérieure, 1996, p. 10-12.

7 voir L. Racine, Remarques sur les tragédies de Racine, Amsterdam, 1752, II, p. 267. 
dans les Essais de Grammaire, et qui adopte un plan. Ce commentaire s'inscrit dans la perspective académique d'une critique "positive". Sur ce point, il est donc très différent du travail de Subligny : l'objectif de D'Olivet, comme celui de l'Académie, est de critiquer ce qui est le plus proche de la perfection, et non pas ce qui appelle la critique par son caractère par trop fautif. L'idée est de partir des textes qui sont les plus remarquables pour, par un petit surcroît de travail grammatical, atteindre une perfection qui aurait alors valeur emblématique. A ce propos, D'Olivet fait sienne la déclaration programmatique de Boileau : "Je voudrois, disoit-il, que la France pût avoir ses Auteurs classiques, aussi bien que l'Italie. Pour cela, il nous faudroit un certain nombre de livres qui fussent declarez exempts de fautes, quant au style"8. C'est pourquoi le commentaire de D'Olivet n'apparaîtra pas aussi tatillon et vétilleux que celui de Subligny : c'est un commentaire intéressant, nuancé, et qui prend au sérieux l'idée d'une amélioration possible de la langue de Racine. Le détail est relevé, mais sans intention polémique. D'Olivet estime d'ailleurs qu'il y a de manière générale dans le théâtre de Racine un "très-petit nombre de fautes". (D'Olivet, 1767 : 255).

Le texte de Racine était-il déjà sacré aux yeux des contemporains de D'Olivet ? Il faut le croire à lire la réponse que lui fit Desfontaines, le plus odieux des commentateurs grammaticaux du $\mathrm{XVIII}^{\circ}$ siècle $^{9}$, prenant le

8 D'Olivet, Remarques sur Racine, Paris, Barbon, 1767, p. 253.

9 voir le Dictionnaire néologique [1726], Amsterdam, Chez Michel-Charles Le Cene, 1748, écrit de manière entièrement 
parti de Racine contre ce qu'il prend pour une attaque en règle dans un : Racine vengé, ou Examen des remarques grammaticales de M. l'abbé D'Olivet sur les oeuvres de Racine, Avignon, 1739. L'idée générale de Desfontaines est de défendre l'exception poétique contre le scrupule grammatical. Dans sa préface, il précise que son intention a été de "soutenir, contre ses opinions [celles de D'Olivet], les privilèges de la langue poëtique, \& défendre les vers de Racine, qu'il a censurez mal a propos". Il reproche ainsi à D'Olivet de n'avoir "mis dans ses remarques aucune distinction entre la Langue Prosaïque \& la Langue Poëtique". Contre la théorie de l'unité grammaticale de la langue, il soutient que "ce qui est faute en prose, souvent n'est pas faute en versification, \& est même un agrément" (Desfontaines, $1739: 2$ ).

Si les parti pris de D'Olivet et de Desfontaines semblent assez opposés, on en lira pourtant une étonnante tentative de synthèse dans les remarques que Louis Racine, le fils de Racine, fit paraître en 1742. Louis Racine suit la méthode de D'Olivet, à savoir celle d'un examen méthodique et linéaire du texte, examen qui accompagne, comme ce n'était pas le cas chez D'Olivet, des remarques d'ordre littéraire faites sur chaque pièce. Il est curieux de voir comment Louis Racine, dont le commentaire est d'ailleurs plus objectif que ne le laisserait supposer son statut familial, va essayer de combiner dans ses remarques l'exigence grammaticale dont il a pris le modèle chez D'Olivet, et la théorie de l'exception poétique dont Desfontaines s'était fait le héraut. De la sorte, et même si

ironique, comme une guirlande de louanges envers les auteurs qu'il ridiculise. 
Louis Racine donne l'impression de n'être jamais complaisant vis-à-vis de tel détail, le texte de son père va se trouver sacralisé du double point de vue de la grammaire et du point de vue de l'"agrammaticalité", si l'on peut dire. Cette entreprise va trouver son couronnement en 1743 avec la grande édition du théâtre de Jean Racine en trois volumes, assortie des remarques de D'Olivet et de Louis Racine, et parue à Amsterdam. Par la suite, les éditeurs successifs : Luneau de Boisgermain (1768), La Harpe (1807) et Geoffroy (1808) vont prendre l'habitude de reproduire ces remarques en note, en en ajoutant certaines de leur cru.

L'histoire de l'examen critique de Racine au XVIII ${ }^{\circ}$ siècle, examen dont l'effet est de renforcer sans cesse davantage la stature de Racine, culmine sans doute avec les positions de Voltaire, et le choix très catégoriquement fait par lui de Racine contre Corneille. Selon Fontanier, "on proposa à Voltaire de faire un commentaire de Racine, comme il faisait celui de Corneille. Il répondit ces propres mots : "Il n'y a qu'à mettre en bas de toutes les pages : beau, pathétique, harmonieux, admirable, etc." ". (Fontanier, 1818 : 31). Le cas de Voltaire nous intéresse tout particulièrement car, comme il a voulu suivre Racine sur ses propres terres, il a été pour ainsi dire l'un des principaux artisans de cette sacralisation -et par voie de conséquence l'une de ses principales victimes... Son idéalisation d'Athalie était très forte. On en veut pour preuve l'amusant détail suivant. On peut consulter à Saint Pétersbourg une édition de Racine ayant appartenu à Voltaire ${ }^{10}$. Il apparaît

10 Racine, Oeuvres, 2 tomes, Paris, Nyon, 1736. Numéro 2854 au catalogue de la Bibliothèque Voltaire à la Bibliothèque 
que ces volumes étaient des volumes d'occasion dont le précédent propriétaire, clairement, était un puriste dans la lignée de Subligny. Ainsi le texte d'Athalie est-il tout parsemé de petits traits et de petites croix destinés à marquer les fautes. On trouve alors de la plume de Voltaire, qui annotait très spontanément et avec beaucoup d'humeur ses livres, cette phrase assassine : "Je voudrais bien savoir quel est l'imbécile qui a défiguré par tant de croix et qui a rempli de fautes le plus bel ouvrage de la langue française". 11

\section{Cas particulier d'Esther et d'Athalie}

Profitons de cette petite anecdote pour noter que, dans l'établissement rétrospectif du "canon grammatical", de l'espèce de "grammaire pratique" ou d'emblème esthétique que le premier $\mathrm{XVIII}^{\circ}$ siècle a fait à partir du théâtre de Racine, les dernières pièces, Esther et Athalie, jouent peut-être un rôle particulier.

Cette spécificité tient tout d'abord au statut écrit des pièces, et au fait qu'elles aient été relues par Racine dans le cadre de leur publication. Ecoutons par exemple ce qu'en dit Louis Racine : "Lorsque dans les Pièces précédentes les Expressions ou les Rimes donnent lieu à quelques critiques, on peut répondre que l'Auteur n'ayant pas mis la dernière main à son Ouvrage, eût peut-être changé ce qui donne lieu à la critique : c'est ce qu'on ne peut plus répondre sur les deux Pièces suivantes. L'Auteur y a mis la dernière main, \& elles ont été imprimées sous ses yeux. Je ne crois pas qu'on

Nationale de Russie. Le premier volume, malheureusement, est manquant. Voltaire possédait aussi l'édition en deux tomes faite à Amsterdam, Henri Schelte, 1713.

11 Ibid., II, p. 423. 
y trouve la moindre négligence dans les Rimes. Lorsqu'on en croit trouver dans les Expressions, il faut bien examiner si l'on ne se trompe point, \& si l'on ne condamne pas trop légérement ce qui n'a paru faute ni à l'Auteur, ni à son sévére Critique, Boileau. " (L. Racine, 1752, II : 217).

D'autre part, ces deux pièces présentent une poétique particulière, où l'intervention de la musique n'est bien évidemment pas indifférente, et a certainement contribué à accentuer, dans l'imaginaire de la langue, la place de l'"harmonie". Ainsi, Esther a été particulièrement admirée par Fontanier pour son style "enchanteur" (Fontanier, 1818 : 571). Les choeurs, surtout, frappèrent les esprits : ils participèrent à l'impression de "sublime", -le terme qui revient le plus souvent dans le commentaire d'Esther. Le commentateur Lefranc, dont une Lettre figure en annexe des remarques de Louis Racine (L. Racine, 1752, II : 440) déclare : "Il m'est venu une pensée en relisant Esther. Ne seroit-ce point la pièce que $M$. Racine s'est attaché à versifier avec le plus de force \& de correction ? J'ose au moins avancer qu'il n'y a pas dans tout ce Poëme un seul vers foible. Quel charme \& quelle énergie de versification ! Que d'expressions neuves ! Que de traits hardis !". Il n'hésite pas à mettre Racine sur le même plan que Virgile, car selon lui, notre auteur satisfait aux trois critères principaux attendus dans l'écriture de la tragédie : harmonie, force, élégance. (Ibid., 441).

De même, dans son Discours de Réception à l'Académie après la mort de Racine (1699), Valincour réserve une place spéciale à un ensemble, l'ensemble formé par : les cantiques, Esther, Athalie. Il parle de "sublimité", 
de "magnificence" (Picard, 1956 : 344). Indiscutablement, il semble qu'il y ait là un nouveau Racine, encore plus extraordinaire s'il se peut que le Racine qu'on connaissait, le Racine dramatique et profane, remplacé à présent par un Racine plus poétique, -et spirituel. Mais c'est peut-être avec Athalie que l'éloge de la langue racinienne atteint son comble, Athalie "appellée par une voix générale, la plus parfaite de nos Tragédies" (L. Racine, 1752, II : 249), ou "l'ouvrage le plus approchant de la perfection, qui soit jamais sorti de la main des Hommes" pour Voltaire ${ }^{12}$. Et on verra dans le choix de l'Académie de l'utiliser comme base à sa grammaire, naturellement, la preuve majeure de cette reconnaissance. Du point de vue de la langue, il semble que les pièces les plus admirées au début du XVIII ${ }^{\circ}$ siècle aient donc été Esther et Athalie. Suit : Iphigénie. Phèdre est souvent jugée magnifique, mais irrégulière. La moins bonne ? Bajazet, peut-être, que bien des commentateurs trouvent "négligée"...

\section{La question du lexique}

Une fois ces premiers éléments posés, notre objectif est à présent d'entrer dans le détail de nos commentaires et d'en proposer une interprétation. Nous allons aborder essentiellement le question du lexique, celle des "tours", ou des alliances de mots, enfin celle des figures.

On a beaucoup commenté la relative pauvreté du lexique de Racine par rapport à celui de Corneille. On se reportera pour cela à l'étude statistique qu'en a faite Charles

12 Voltaire, epistre en tête d'Oreste, The Complete Works of Voltaire, Voltaire Foundation, vol. 31A, 1992, p. 405. 
Bernet $^{13}$. Y a-t-il là un "ascétisme" sous influence janséniste ? Un choix individuel à relier avec une conception dramatique ? Une tendance générale de l'époque ? Sans doute ne faut-il pas négliger la part consciente, le choix esthétique, le parti pris d'écriture : mais probablement aussi faut-il prendre en compte les contraintes de plus en plus draconiennes qui s'imposent à l'auteur de tragédies soumis l'époque de Racine au regard de grammairiens toujours plus exigeants. A y regarder de près, il semble qu'insensiblement, et souvent pour des raisons diverses, un très grand nombre de termes se soient trouvés l'un après l'autre exclus de la diction tragique. De la sorte, de Mairet à Racine, l'histoire de la tragédie classique française se signale par une constante déperdition dans la palette lexicale. On a peine à imaginer, aujourd'hui, l'ampleur de cette chasse aux termes qui a frappé le théâtre classique. Certains des termes que Voltaire incrimine chez Corneille nous étonnent : le terme "civilité", par exemple14, ou le verbe "clore" (Voltaire, 1974 : 714). De façon générale, on peut dire que ces termes se répartissent en plusieurs registres.

Le premier registre concerné, et le plus important sans doute, est celui des termes concrets, particulièrement ceux décrivant les réalités physiques, corporelles.

Dans son commentaire de Phèdre, L. Racine se permet de citer à plusieurs reprises des passages de la Phèdre de Garnier, écrite, dit-il, "lorsque nous ignorions

13 Ch. Bernet, Le Vocabulaire des tragédies de Racine : analyse statistique, Paris, Champion, 1983.

14 Voltaire, Commentaires sur Corneille, The Voltaire Foundation, 1974, p. 738. 
encore ce que c'étoit que Tragédie", "pour faire connoître, dit-il les changemens arrivés à notre Langue"(L. Racine, 1752, II : 130). Il est vrai que certains passages de cette pièce sont particulièrement chargés de termes et de constructions rendus impossibles plus tard dans l'écriture tragique :

La Phèdre de Garnier était très physique :

"[...] L'Amour consomme enclos

L'humeur de ma poitrine \& desseche mes os.

Il rage en ma moelle, \& le cruel m'enflâme

Le coeur \& les poumons d'une mortelle flâme."

Mais comment ne pas reconnaître, dans ces vers, un aspect de l'élocution si particulière, les termes concrets en moins, avec laquelle la Phèdre de Racine énoncera sa passion ... "Les ennemis de notre Poëte l'accusoient de piller les anciens Poëtes François", dit L. Racine (1752, II : 135). Racine, en effet, ne pouvait pas employer le mot poitrine. Selon Vaugelas, que cite L. Racine, poitrine doit être retiré de la tragédie sous le prétexte qu'on dit poitrine de veau. Pour Subligny, de même, il y a beaucoup de impur, inceste, adultère, chaste, etc., dans Phèdre, termes "qui ne doivent point entrer dans des vers" (Granet, 1740 : 404). Mais si elle ne parle pas de poitrine, Phèdre dit bien, malgré tout :

"Ce n'est plus une ardeur dans mes veines cachée"...

"Nous n'osons nommer les nerfs, \& nous nommons élégamment les veines", dit L. Racine (L. Racine, 1752, II : 132). Pour lui, donc, il n'y a pas beaucoup de cohérence dans ce système d'exclusions. D'ailleurs, ajoute-t-il après avoir noté qu'estomac "passait", de même, dans un passage de la Rodogune de Corneille, "tous ces mots proscrits de notre style noble, sont très nobles dans la Langue Latine". (Ibid.). 
Le deuxième registre est celui des termes décrivant des relations sociales ou familiales. Pour Voltaire, ces termes doivent être exclus de la tragédie. Chez Corneille, il relevait -citons-en quelques-uns au hasard- des maîtresse (Voltaire, 1974 : 740), bourgeois (Ibid., 738), mari, père (Ibid., 766), qui le choquaient... Et Louis Racine peut bien s'étonner de trouver un gendre dans Iphigénie... ("Il vient en m'embrassant de m'accepter pour Gendre", Iphigénie, III, 2). "Nous avons des mots qui n'entrent point dans le stile Poëtique, sans qu'on en puisse dire la raison.", conclut-il perplexe (L. Racine, 1752, II : 40). Et il remarque que, chez Corneille, beau-père et gendre "ont une grandeur que n'auraient point les noms de César \& de Pompée" (Ibid.). Y a-t-il là un paradoxe poétique ? L. Racine conclut : "C'est par l'art de placer les mots, qu'un habile Ecrivain les annoblit".

Le troisième registre est celui des "locutions familières", celles qu'on emploie dans la conversation. A partir d'Alexandre, dit Fontanier, Racine fait disparaître de son style les locutions familières qui entachaient le style de Corneille (Fontanier, 1818 : 74). Ce qui est paradoxal ici, c'est que Corneille s'était précisément fait une originalité et un mérite d'avoir introduit ces locutions familières au théâtre, alors que celui-ci était envahi, à son époque, par des tournures rhétoriques stéréotypées ${ }^{15}$. Dans l'examen de Mélite, datant de 1660, Corneille attribuait à cette particularité le succès de ses premières pièces : "La nouveauté de ce genre de comédie, dont il n'y a point

15 voir G. Conesa, "Le style de la comédie", Littératures classiques, $\mathrm{n}^{\circ} 28$, automne 1996, p. 95-111. 
d'exemple en aucune langue, et le style naïf qui faisait une peinture de la conversation des honnêtes gens, furent sans doute cause de ce bonheur surprenant qui fit alors tant de bruit"16. Toutefois, il pressentait bien que l'élévation constante du style au théâtre allait bientôt faire mal considérer cette façon d'écrire : "Vu que ma façon d'écrire était simple et familière, la lecture fera prendre mes naïvetés pour des bassesses". 17

Dans chacun de ces registres, on notera que la langue de Racine, finalement, enfreint quelque peu les règles alors en train de s'établir. Rétrospectivement, donc, on peut imaginer que certaines particularités de sa langue ont placé les commentateurs dans l'embarras : ils ne savent plus ce qui doit être admis et ce qui ne doit pas l'être, ce qui est trop concret, ce qui est trop familier, etc. Tout paraît suggérer que ces commentateurs ont eu en tête, tout autant qu'un imaginaire lexical de type général et dominé par une tendance à l'abstraction, la vision d'un point d'équilibre que la langue se devait d'atteindre, et que le genre tragique aurait pour mission d'illustrer au moyen de la noblesse de ses sujets et de la rigueur de ses contraintes. Toutefois, il est clair qu'il s'agissait d'un point d'équilibre difficile à appréhender hors de l'expérience d'écriture elle-même. Aussi l'autorité de Racine a-t-elle paradoxalement servi à fixer les contours de cette configuration imaginaire, amenant les commentateurs à souligner du même coup, d'une manière qui n'est pas sans mettre en danger la validité de la discipline grammaticale, le rôle du contexte dans l'appréciation des mots, et celui du talent personnel de

16 Corneille, Oeuvres complètes, Paris, Le Seuil, 1963, p. 28.

17 Ibid. 
l'écrivain dans la manière de les "placer". Le fait que l'esthétique lexicale de Racine ne soit pas radicale, qu'elle porte à l'état de traces de nombreuses marques d'époques antérieures dont les théoriciens voulaient se débarrasser, place ces derniers dans une position malaisée dans leur débat personnel avec l'imaginaire linguistique.

L'un dans l'autre, par conséquent ${ }^{18}$, il est clair que Racine s'est trouvé confronté à une diminution des termes acceptés dans le style tragique dont on peut se demander s'il l'a ou non reçue favorablement. On peut imaginer que cette diminution l'a exposé à plus d'une difficulté dans l'écriture de tel ou tel passage, qu'elle la amené à faire des choix, à prendre des risques. Mais ce qui est le plus frappant, en définitive, c'est que souvent ses choix, s'ils paraissaient problématiques au départ, ont fini par s'imposer, et par stabiliser tant soit peu cette dérive à grande allure vers un lexique tragique très pauvre, très pur, et très abstrait, qui se mettait en place à la fin du $\mathrm{XVII}^{\circ}$ siècle.

\section{Les alliances de mots, les "tours".}

Si Racine, comme on le voit, se trouvait confronté dans son écriture à une diminution dramatique du lexique utilisable, il ne devait pas trouver dans les néologismes, à l'instar de ses aînés, un moyen d'élargir tant soit peu sa palette : sur ce point, les grammairiens de son époque sont unanimes et se rangent à l'avis de Vaugelas : "il ne faut

18 Et plus encore si l'on tient compte de l'élimination des licences poétiques, telle cet emploi de croître transitif dans Esther, par exemple ("Que ce nouvel honneur va croître son audace"), que l'Académie ne laisse plus passer (voir L. Racine, II, 224). 
jamais faire de mots" 19 . Pourquoi ce refus si obstiné des néologismes, cette censure, faite même aux plus grands écrivains, de toute invention lexicale ? D'Olivet a un point de vue original et pragmatique. Pour lui, il faut autant que faire se peut éviter de recourir à des mots "qu'on a vu naître", dit-il, parce que ce serait faire trop dépendre la survie de son oeuvre de la survie de ces termes. Un écrivain judicieux, dit-il, donnera "aux mots le temps de s'établir assez bien pour n'avoir plus rien à craindre de la fortune". (D'Olivet, 1767 : 276). Mais sans doute y a-t-il là aussi une trace majeure du figement de l'imaginaire linguistique à l'époque classique.

Sur ce point délicat des néologismes et de l'usage, Racine se sera montré un écrivain judicieux. Il sera donc peu repris sur la question des "mots". Louis Racine défend son père globalement contre ce possible reproche : "il ne faisoit point de mots", dit-il (L. Racine, 1752, II : 221). De même D'Olivet déclare : "il ne s'y trouve pas un mot nouveau, c'est à dire, un de ces mots qui se faisoient de son temps ; comme il s'en est toujours fait, \& comme il s'en fera toujours." (D'Olivet, 1767 : 276). Encore lui arrivera-t-il d'être repris occasionnellement sur un terme dont l'usage ne s'est pas tout à fait imposé : l'adjectif respectable, par exemple, qu'on trouve dans ce vers d'Esther, et qui nous semble aujourd'hui bien anodin :

"Un éclat qui le rend respectable aux Dieux mêmes" ( Esther, II, 5).

Respectable : ce mot, selon Richelet, "de nouvelle fabrique, [et dont] on ne se sert pas encore librement" ; ce

19 Vaugelas, Remarques, éd. J. Streicher, Genève, Droz, 1934, Préface, XI. 
mot, selon Louis Racine, qui à la différence de respect, n'est pas un latinisme direct. (L. Racine, 1752, II : 222).

Le problème de la langue de Racine n'est donc pas dans les mots : il est ailleurs. Il se trouve résumé par cette phrase percutante de Louis Racine : "on s'aperçut que le poète, en inventant, non des mots, mais des alliances de mots, mais des tours de phrase, faisait, pour ainsi dire, une langue nouvelle" (cité par Fontanier, 1818 : 101). On comprend en effet que, face à de telles contraintes dans le choix du lexique, l'écrivain se soit trouvé amené à travailler sur d'autres procédés : des alliances de mots, des "tours", quitte à ce que les mots choisis, en eux-mêmes, soient assez ordinaires. A vrai dire, la critique des "tours", incontestablement, joue un rôle particulier dans la réception du texte racinien. Les tours possèdent en effet une iconicité de type à la fois syntaxique et sémantique susceptible de donner de la langue autant de physionomies momentannées, de petites images circonscrites, qui installent un usage, définissent des contours, créent des passages obligés. Ils figent des constructions autant que des effets de sens et, ce faisant, aliènent véritablement l'expression. Le "génie" entier de la langue s'y trouve impliqué. C'est pourquoi de très nombreuses remarques, tant de l'Académie que de D'Olivet, que de Louis Racine leur sont consacrées.

Il est très frappant de constater que, dans l'examen qui va être fait du texte racinien, les tours vont systématiquement être sortis de leur contexte : et en effet, comme ils sont jugés suceptibles d'être représentatifs de la langue dans sa totalité, ils doivent, en eux-mêmes, quel que soit leur emploi, être individuellement "français". Ainsi, 
bien souvent, l'exercice favori des critiques consiste à séparer, dans l'analyse d'un micro-syntagme, le commentaire de la construction, de l'activation particulière d'un sens, de la congruence sémantique ou syntaxique des termes, etc., -de l'entourage dans lequel ce syntagme se trouve. Il en résulte presque systématiquement une impression d'étrangeté qui motive un choix : acceptation ou exclusion.

Il semble, comme nous le disions, que ce soit avec Andromaque que la nouveauté de la méthode racinienne, et donc son caractère linguistiquement inacceptable, se soit déclarée. On en veut pour preuve la déclaration spectaculaire de Subligny dans la préface de sa Folle Querelle : "Si l'on veut se donner la peine de lire l'Andromaque avec quelque soin, on trouvera que les plus beaux endroits où l'on s'est écrié \& qui ont rempli l'imagination de plus belles pensées, sont toutes expressions fausses ou tronquées qui signifient tout le contraire ou la moitié de ce que l'Auteur a conçu lui-même, $\&$ que parce qu'un mot ou deux suffisent à faire souvent deviner ce qu'il veut dire, \& que ce qu'il veut dire est beau, l'on y applaudit, sans y penser, tout autant que s'il étoit purement écrit \& entièrement exprimé". (Racine, 1999 : 259). Il donne comme exemple, entre autres : "apaiser sa rigueur", "venger ses mépris", etc (Ibid., 260).

Selon Louis Racine, son père corrigea certaines des "fautes" relevées par Subligny, mais "laissa néanmoins subsister certains tours nouveaux, que Subligny mettait au nombre des fautes de style, et qui ayant été approuvés depuis comme des tours heureux, sont devenus familiers à 
notre Langue."(Racine, 1999 : 1133). Tout de même, dit L. Racine, on a approuvé :

"Mais de faire fléchir un courage inflexible" dans Phèdre (II, 1), mais on a "ri" au :

"Mais on n'efface point des traits ineffaçables" dans l'Electre de Longepierre (L. Racine, 1752, II : 134). Pourquoi donc les alliances de mots inédites de Racine ont-elles réussi à s'imposer ? "Tout Ecrivain ne sait pas faire approuver ses Expressions", conclut L. Racine (Ibid.), qui se demande par ailleurs si ce n'est pas de l'examen trop local d'une phrase qu'on fait naître la difficulté : "Le sens se présente toujours si naturellement dans les Vers de ces Tragédies, qu'on ne fait pas quelquefois attention à la hardiesse du tour." (L. Racine, 1752, II : 36). Pour lui, la caractéristique du théâtre de son père, ce sont précisément ces tours un peu bizarres dans le détail, ces constructions irrégulières, ces inversions parfois osées, dont aucune obscurité ne résulte : ce thème forme le leit-motiv de son commentaire de Phèdre, par exemple (L. Racine, 1752, II : 129). Les uns, tel D'Olivet, attribuent ce caractère à une singularité individuelle : "Mais des termes les plus communs, il avoit le secret d'en faire un langage qui lui appartient, \& n'appartient qu'à lui." (D'Olivet, 1767 : 284). Mais les autres, tel La Mothe cité par L. Racine dans ses Réflexions sur la poésie y voient de plus comme un appel du génie profond de la langue : "Il s'était fait, par une intelligence particulière, une langue qui n'appartenait qu'à lui seul. Combien d'alliances de mots, inusitées jusqu'à lui, dont on n'a presque pas aperçu l'audace ? Ce qu'il inventait semblait plutôt manquer à la langue que la violer." Singularité individuelle et obéissance au génie de la langue se confondent alors dans une même idéalisation. 


\section{La réticence face aux figures}

La réticence face aux figures fait partie des refrains de l'imaginaire linguistique français, de Bouhours à Rivarol, lequel déplore ce perpétuel "mensonge" du langage. Pour Bouhours, la métaphore est véritablement le noeud de la différence qui sépare notre langue de la plupart des autres langues romanes. "Pour la metaphore, elle ne s'en sert que quand elle ne peut s'en passer ; ou que les mots metaphoriques sont devenus propres par l'usage. Sur tout, elle ne peut supporter les metaphores trop hardies : \& nous ne sommes plus au temps du zenit de la vertu, du solstice de l'honneur, \& de l'apogée de la gloire."20. Ainsi, pas plus que les néologismes ou les termes concrets ou familiers, Racine ne pourra employer les métaphores voyantes de l'écriture poétique baroque. Les métaphores ponctuelles éblouissent, mais cela n'est pas considéré comme une qualité. Ainsi, à propos du vers de Mithridate :

"Au-dessus de leur gloire un naufrage élevé" (Mithridate, II, 4, 33),

le P. Du Cerceau écrit-il : "Ces expressions figurées ont d'abord quelque chose qui éblouit, \& l'on ne se donne pas la peine de les examiner, parce qu'on les devine plutôt qu'on ne les entend : mais quand on y regarde de près, on est tout surpris de ne trouver qu'un barbarisme brillant dans ce qu'on avoit admiré" 21 .

C'est ainsi que le récit de Théramène dans Phèdre, pour Laharpe, "brille d'un luxe de poésie quelquefois

20 D. Bouhours, Entretiens d'Ariste et d'Eugène, Paris, A. Colin, 1962, p. 35.

21 P. Du Cerceau, Réflexions sur la Poësie Françoise, Amsterdam, Bernard, p. 254. 
déplacé" (cité par Fontanier, 1818 : 560). Effectivement, on y trouve de l'"harmonie imitative" ("l'essieu crie \& se rompt, "qui fait entendre le cri de l'essieu" pour L. Racine, 1752, II : 244), et surtout cette hypotypose :

"Le flot, qui l'apporta, recule épouvanté".(V, 6)

critiquée par D'Olivet, et que Desfontaines, dans une petite fiction où il fait dialoguer Théramène lui-même avec D'Olivet, s'efforcera de sauver.(Desfontaines, 1739 : 105).

Faut-il néanmoins écrire absolument sans métaphores ? En prose, pour les rendre plus acceptables, Bouhours préconisait d'user de tours d'accompagnement tels que : "si je puis dire"..., destinés à atténuer l'impression de choc. Il est clair qu'en poésie, cette solution n'était pas possible. Un autre procédé s'offrait : la métaphore continuée, autrement dit l'emploi de termes (souvent deux), qui suivent la métaphore, et qui l'incorporent pour ainsi dire au texte. En contre-exemple de quelques métaphores trop osées chez Racine, Fontanier cite ce vers de la Mérope de Voltaire (V, 6), plus conforme à son idéal :

"De ces flots confondus le flux impétueux"... (Fontanier, $1818: 618$ ).

Pour lui, dans Alexandre, d'ailleurs, il y avait quelques métaphores "soutenues", qui annonçaient un grand poète (Fontanier, 1818 : 47). De même, L. Racine admire un passage d'Iphigénie (Iphigénie, V, 2 ; L. Racine, 1752, II : 40) du fait que "tous les mots répondent à la métaphore". "Iphigénie parle de moissons, de gloire, elle dit des mains vaillantes, pour les recueillir, \& un champ, qui sera stérile s'il n'est arrosé"... Ainsi pouvait se mettre en place une sorte de langage codé, une phraséologie tout à la fois 
"poétique" et reconnaissable dans le "tour", qui, de loin en loin, élevait le langage tragique sans l'éloigner inconsidérement de sa mission de représentativité. Dans la "métaphore continuée", une sorte de logique dans l'alliance des termes s'impose au jugement, qui peut faire croire en un caractère linguistiquement contraint de l'expression. Dans les tragédies de Voltaire, lequel s'inspirera fortement du modèle de la métaphore continuée, l'iconicité de ce type de phraséologie éclatera, et s'avèrera destructrice de la recherche expressive...

Qualifiée par les grammaires de l'époque de "figure de construction", à cheval entre les problèmes de figures et les problèmes de syntaxe, l'ellipse est encore un exemple de ces physionomies locales voyantes qui suscitent la réaction des commentateurs raciniens. La plus célèbre des ellipses de Racine est sans doute celle-ci, tirée d'Andromaque :

"Je t'aimais inconstant, qu'aurais-je fait fidèle ? ".

Elle sera abondamment commentée par D'Olivet (D'Olivet, 1767 : 376). Celui-ci en relève d'autres exemples, et conclut : "Toutes ces phrases, au moyen de l'Ellipse [il entend par là sa théorie de l'ellipse], rentreront dans les règles de la Syntaxe ordinaire"(D'Olivet, 1767 : 264). Toutefois, il est notable que Desfontaines la défende et la trouve "admirable" (Desfontaines, 1739 : 23). D'ailleurs, il déclare : "L'ellipse est une figure autorisée, \& qui fait quelquefois une grande beauté, placée à propos." (Desfontaines, 1739 : 57). C'est pourquoi il reproche à D'Olivet de vouloir "la proscrire à jamais" (Ibid.). 


\section{Racine sauvé}

Racine, épluché, Racine décortiqué, Racine contesté, mais Racine sauvé, finalement. Pourquoi ?

Il est un paramètre avec lequel il faut compter, et qui nous surprend un peu aujourd'hui, c'est celui de l'insécurité linguistique. Dans Les Deux Ages du Goût et du Génie français sous Louis XIV et sous Louis XV de M. de la Dixmerie (1749), l'auteur propose une petite fiction où il suit le Génie au hasard de ses déplacements dans des palais. Les grands auteurs s'avancent, se présentent, lisent des extraits de leurs oeuvres, et motivent de la sorte quelques jugements. Ainsi, à propos de Racine, lit-on qu'on trouve chez lui "une harmonie, une élégance inaltérable". Suit cette phrase : "Il fit passer dans ses écrits, dit-il, toute la magnificence d'une Langue qui ne faisait que de naître, \& qu'avant lui on croyait barbare \& pauvre." (Dixmerie, 1749 : 218). Cette dernière phrase peut nous étonner aujourd'hui ; elle nous révèle du moins à quel point subsistait encore à l'époque de Racine une manière de complexe d'infériorité, en premier lieu à l'égard des langues anciennes, mais aussi à l'égard de quelques langues modernes comme l'italien, dont il convenait à tout prix de se débarrasser. Quel est donc le sens de l'entreprise de D'Olivet ? Ce n'est pas de faire apparaître le français comme une langue difficile, c'est au contraire de persuader le lecteur que c'est une langue qui est susceptible de perfection, et que cette perfection est accessible (D'Olivet, 1767 : 388).

D'ailleurs, quelles sont les valeurs essentielles qui sont exaltées dans la langue de Racine ? Ce sont les valeurs de sublimité et de simplicité-valeurs sécurisantes, qui sont posées comme valeurs générales de la langue, à la portée de 
tout un chacun, et qui délivrent donc celui qui leur obéit de la fatigante recherche d'un talent individuel.

Car il n'y a pas un mince paradoxe à voir comment, finalement, la sacralisation prgressive du texte de Racine s'est faite de manière à ce que se trouvent cumulés l'éloge de la régularité et l'éloge de l'irrégularité. L'éloge de la régularité, on peut dire que c'est D'Olivet qui l'a assumé. Son point de vue est un point de vue parfaitement "classique" : il se nourrit des idées de Bouhours et de Boileau, Boileau dont d'ailleurs une pleine page de citations est fournie pour défendre l'idée que "sans la langue", on ne peut pas être un bon écrivain (D'Olivet, 1767 : 327). Pour D'Olivet, de toute manière, depuis Vaugelas, la langue française ne varie plus que sur des détails (D'Olivet, 1767 : 334).

L'irrégularité est défendue par Desfontaines. L'essentiel de sa défense de Racine contre les remarques de D'Olivet, finalement, tourne autour de la possibilité ou non de privilégier l'exception poétique face à la régularité grammaticale. Reprenant des arguments qui avaient été ceux de Marie de Gournay contre Malherbe ${ }^{22}$, il est d'avis que oui, il faut placer les textes de poésie en dehors du scrupule grammatical. Il s'agit non seulement de sauver l'inspiration poétique, souffle d'émotion qui ne peut se permettre de se voir sans cesse entravé par des minuties de grammaire, mais de défendre l'idée d'un caractère linguistiquement différent de la langue utilisée en poésie. Cette théorie, bien entendue, ne satisfaisait pas D'Olivet,

22 Marie de Gournay, Les Advis, ou les Présens de la Demoiselle de Gournay, $3^{\circ}$ éd., Paris, Jean du Bray, 1641. 
car, disait ce dernier, "notre langue ne ressemble pas à quelques autres où la Poësie \& la Prose font, pour ainsi dire, deux langages différents. Ce n'est pas que le François ne connoisse qu'un même style pour ces deux genres d'écrire. Mais les différences qui doivent les caractériser, ne sont pas grammaticales pour la plupart. " (D'Olivet, 1767 : 255).

Le plus curieux est que la critique que Desfontaines a adressée à D'Olivet, malgré tout, a porté, puisque L. Racine en tient compte, et peut se donner les gants de faire intervenir, à doses savamment alternées, le souci de régularité et l'excuse au génie. Ainsi, l'exception elle-même est récupérée, et l'impact de l'émotion poétique, le sentiment d'inspiration, sont mis au service d'une théorie dont l'objectif est malgré tout l'assentiment linguistique et la soumission au génie de la langue. On notera que cet étonnant paradoxe, qui trouve à l'épreuve du texte racinien l'occasion de s'affermir et de se constituer une manière de vérification pratique, avait déjà fait l'objet de postulations théoriques dans l'oeuvre de Vaugelas. Et, sur ce point, Vaugelas est approuvé par D'Olivet (D'Olivet, 1767 : 377). A vrai dire, la théorie de l'agrammaticalité élégante est peut-être celle, plus encore que le purisme, ou les théories du "régulièrement écrit", qui est le plus à même de faire d'un texte littéraire, ou poétique, un emblème linguistique. D'Olivet reconnaît d'ailleurs avoir fait le choix d'un poète, pour sa critique grammaticale, en raison de la valeur emblématique plus grande de la poésie : "J'ai préféré un Poëte, parce qu'il me semble que d'excellens vers se font lire \& relire plus volontiers, qu'une prose également bonne en son genre. " (D'Olivet, 1767 : 256). Pour lui, Racine en 
poésie est plus proche de la perfection linguistique que n'importe quel auteur de prose. (Ibid.) Et on trouve dans son commentaire de nombreux passages où transparaît l'aveu d'une sorte d'impuissance devant un pouvoir qui transcende les efforts de la critique. Citons cette phrase touchante et significative : "J'aurois eu souvent de ces riens à observer dans Racine : mais que m'arrive-t-il ? Après un moment de réflexion sur l'espèce de faute qui m'arrêtoit, je retourne à ma lecture; \& bientôt cette belle simplicité, cette douce harmonie, cette élégance, cette éloquence, qui sont le ton dominant, viennent à me frapper de façon que je finis par être honteux d'avoir eu la tentation de critiquer." (D'Olivet, $1767: 364$ ).

Passées les premières disputes, de Subligny à la "revanche" de Desfontaines sur D'Olivet, l'histoire de la critique linguistique sur Racine se mue donc bientôt, les remarques de son fils Louis aidant, consolidées à leur tour par la somme de Fontanier, en une histoire de la sacralisation de son texte. Ce phénomène de sacralisation du texte par rapport à la critique, qui était assez inconnu du $\mathrm{XVII}^{\circ}$ siècle, se lit bien dans l'attitude d'un Voltaire plaçant d'un côté, les écrivains parfaits, intouchables, géniaux, et de l'autre côté les commentateurs, très dévalorisés. Dans le Siècle de Louis XIV, il représente Bouhours assis dans un coin en train de corriger fébrilement toutes les fautes des grands écrivains, pendant que ceux-ci apparaissent dans toute leur gloire. Racine, pour lui, ne peut plus être touché. Qui pourrait, à vrai dire, être tenté de le faire : au milieu du $\mathrm{XVIII}^{\circ}$ siècle, la langue de Racine n'est plus celle de tous les jours : l'intention normative à son égard disparaît. La langue de Racine devient la figure d'un génie transcendant 
qui, tout autant que celle d'une sécurité linguistique, contient la promesse d'un accomplissement affranchi des menaces du temps. Beaucoup font la remarque que l'usage racinien s'est en quelque sorte figé. L'observation que, dans son ensemble, la langue de Racine semble ne pas avoir beaucoup bougé, vient alors rejoindre le rêve que la langue française en général atteigne une sorte d'état stable, hors du temps. Pour Voltaire, un seul terme du lexique racinien était sorti d'usage : il s'agit curieusement du mot ressentiment, dans Bérénice (cité par D'Olivet, 1767 : 283). Tout le reste était inchangé. D'Olivet ne pouvait que s'étonner de ce phénomène : "Car enfin, à remonter du jour où j'écris ceci jusqu'au temps où parurent les premières Tragédies de Racine, nous avons un siècle révolu. Voit-on ailleurs cette pureté inaltérable, \&, si j'osois parler ainsi, cette fraîcheur de style, toujours la même au bout de tant d'années ?" (Ibid.).

C'est que la langue racinienne est sortie de la problématique de l'usage. Cette sortie signe la grande ambiguïté de l'imaginaire linguistique classique, car, sur quoi, en réalité, se fonde cet imaginaire ? En partie sur les règles, certes, sur ce qu'on appelle alors "les principes" : mais aussi beaucoup sur un assentiment mystérieux, obéissant aux lois de l'"oreille", à une sorte de voix secrète que le bon écrivain, le bon locuteur, le bon connaisseur de sa langue, est censé entendre derrière l'"usage". "On n'a de conseil à prendre que de l'usage, dans une matière où le raisonnement n'entre pour rien", dit finalement D'Olivet. (D'Olivet, 1767 : 320). C'est pourquoi Racine, en dépit des discussions, ne sera finalement sauvé que par son seul usage... La sacralisation de son texte a ceci de fascinant 
qu'elle est une sacralisation fondée sur l'usage, mais pensée sous les couleurs du mythe, autrement dit d'un monde sans usage.

Gilles Siouffi

Université Paul Valéry, Montpellier III

\section{Bibliographie :}

DESFONTAINES, Racine vengé, ou Examen des remarques grammaticales de M. l'abbé D'Olivet sur les oeuvres de Racine, Avignon, 1739.

DIXMERIE, M. de La, Les Deux Ages du Goût et du Génie français sous Louis XIV et sous Louis XV, Paris, La Harpe, 1749.

D'OLIVET, Remarques sur Racine, in Remarques sur la langue françoise, Paris, Barbon, 1767.

FONTANIER, P., Etudes de la langue française sur Racine, ou Commentaire général et comparatif sur la diction et le style de ce grand classique, d'après l'abbé D'Olivet, l'abbé Desfontaines, Louis Racine, Voltaire, l'Académie, Luneau de Boisgermain, Laharpe, et Geoffroy ; pour servir comme de cours pratique de langue française, et suppléer à l'insuffisance des grammaires, surtout en ce qui concerne l'application des principes, Paris, Belin, Le Prieur, 2 volumes, 1818.

-GRANET, Recueil de dissertations sur plusieurs tragédies de Corneille et Racine, Paris, Gissey, 1740, 2 volumes. 
-PICARD, J., Corpus Racinianum. Recueil inventaire des textes et documents $d u X V I I^{\circ}$ siècle concernant Jean Racine, Paris, Les Belles Lettres, 1956.

-RACINE, J., Oeuvres complètes, I, éd. G. Forestier, Paris, Gallimard, Bibliothèque de la Pléiade, 1999.

-RACINE, L., Remarques sur les tragédies de Jean Racine, Amsterdam, Marc Michel Rey, et Paris, Desaint et Saillant, 1752, 2 volumes.

-VOLTAIRE, Commentaires sur Corneille, in The Complete Works of Voltaire, tomes 53-55, The Voltaire Foundation, Theodore Besterman, Thorpe Mandeville House, Banbury, Oxfordshire, 1974. 